\title{
Acute Lymphoblastic Leukemia in Adolescents and Young Adults
}

\author{
Josep-Maria Ribera \\ Clinical Hematology Department \\ Institut Català d'Oncologia \\ Hospital Universitari Germans Trias i Pujol \\ Institut de Recerca contra la Leucemia Josep Carreras \\ Universitat Autònoma de Barcelona \\ Spain
}

\section{Introduction}

The development of effective therapy for children with acute lymphoblastic leukemia (ALL) is one of the greatest successes of clinical oncology, with long-term survival achieved in about $90 \%$ of children 1-10 years of age (Pui et al 2008, Pulte et al 2008). However, cure rates for adults with ALL remain relatively low, at only 40\%-50\% (Gokbuget et al 2009, Larson et al 2008). In the last two decades significant improvements in survival for older adolescent and adults (aged 15-59 years) with ALL have been observed, being especially evident in patients aged $15-19 \mathrm{yr}$.

Age is a continuous prognostic variable in ALL with no single age at which prognosis deteriorates markedly. Within childhood ALL populations, older children have shown inferior outcomes (Pulte et al 2009, Smith et al 2010) while younger adults have shown superior outcomes among adult ALL patients (Moorman et al 2010, Juliusson et al 2010). The definition of the age range that encompasses the adolescent and young adults (AYA) patient is controversial, ranging from 15 to $21 \mathrm{yr}$ in some studies, from 15-30 yr in others or even from 15 to $40-45 \mathrm{yr}$ in others. This chapter will focus on the results of treatment of AYA with ALL.

\section{Clinical and biologic characteristics in adolescents and young adults}

The incidence of ALL decreases with age, ranging from 9-10 cases/100,000 persons/year in childhood (representing $30 \%$ of all cancers) to $1-2$ cases/100,000 persons/year in adults. In adolescents the incidence is 3 cases/100,000 persons/year and represents $6 \%$ of all cancers at that age (Bleyer et al 2006).

Several clinical and biologic characteristics of ALL are age-dependent. In this sense, T-ALL is more frequent in AYA (25\%) than in children (10-15\%) or in older adults (Pullen et al 1999). However, the most important differences lie in cytogenetic and molecular characteristics. For example, there is a lower frequency of hyperdiploidy $>50$ chromosomes or ETV6/RUNX1 (previously TEL/AML1) in AYAs (20\%) compared to children 1 to 9 years of age (>30\%) (Moorman et al 2010). In addition, the frequency of MLL (myeloid-lymphoid leukemia or mixed lineage leukemia gene, usually associated with 11q23) rearrangements in 
non-infant ALL increases with age, being infrequent in children 1-9 $\mathrm{yr}$ of age and about $6 \%$ in adults (Moorman et al 2010). Regarding structural changes, there is a progressive increase in the frequency of $\mathrm{t}(9 ; 22)(\mathrm{q} 34 ; \mathrm{q} 11)$ or $B C R-A B L$ rearrangements, ranging from less than $3 \%$ in children under $18 \mathrm{yr}$. to $6 \%$ at ages $18-25$ and to $15-20 \%$ at ages $25-35 \mathrm{yr}$, and to more than $30 \%$ over the age of $35 \mathrm{yr}$ (Secker-Walker et al 1991). Finally, in recent studies, adolescents were more likely to have detectable minimal residual disease (MRD) during or at the end of remission induction (Pui et al 2011).

In summary, with increasing age there is a progressive increase in the frequency of subsets of ALL patients with genetic abnormalities associated with poor prognosis and these changes have already become evident in AYA patients.

As far as host factors are concerned, several features are observed in less young patients, being responsible for increased treatment toxicity. They include differences in the metabolism of chemotherapeutic agents, depleted marrow reserve and increased extramedullary toxicity. All these issues increase the frequency of life-threatening infections, organ failure, and treatment delays and dose reductions in planned chemotherapy.

\section{Which is the best treatment strategy? Pediatric-based vs. adult-based treatments}

\subsection{Retrospective comparative studies}

A number of comparisons of the clinical outcome of adolescents enrolled in adult and pediatric clinical trials have resulted in interesting observations about the appropriate treatment strategy for prospective studies in AYA. Several retrospective reports have shown that adolescents (15- $20 \mathrm{yr}$.) and young adults treated by adult oncologists or hematologists with adult ALL protocols have poorer outcomes than similarly aged patients treated by pediatricians with pediatric protocols, despite having similar biologic characteristics of the disease (Boissel et al 2003, de Bont et al 2004, Testi et al 2004, Hallbook et al 2006, Schroeder et al 2006, Ramanujachar et al 2007, Lopez-Hernandez et al 2008, Stock et al 2008, Al-Khabori et al 2010).

The first study in which such different outcomes were reported was performed in France (Boissel et al 2003). A comparison of AYA aged 15-20 yr. treated with the pediatric-based protocol FRALLE-93 $(n=77)$ with patients of the same age and comparable clinical and biologic characteristics of ALL who received the adult-based protocol LALA-94 ( $\mathrm{n}=100)$ showed a complete remission (CR) rate of $94 \%$ vs. $83 \%$. After a median follow-up of $3.5 \mathrm{yr}$, the event-free survival (EFS) probabilities were $67 \%$ vs. $41 \%$ at 5 years. Multivariate analysis showed an independent influence of the protocol on the outcome. The differences in the drugs employed and, especially in the dose-intensity, could explain the better results of the FRALLE-93 protocol. In this protocol the cumulated dose of prednisone was five-fold higher, the vinca alkaloids three-fold and the asparaginase 20-fold higher than in the LALA94 study. In addition, in the FRALLE-93 study the dose of prednisone in induction was higher and asparaginase was also given in this period, in contrast with the LALA-94 trial. Moreover, the time interval between $\mathrm{CR}$ and post-remission therapy was 2 days in FRALLE93 vs. 7 days in the LALA-94 study.

The North-American Cancer and Acute Leukemia Group B (CALGB) and the Children's Cancer Group (CCG) performed a retrospective comparison of presenting features, planned treatment, CR rate, and outcome of 321 AYA aged 16 to 20 years who were treated on consecutive trials in either the CCG or the CALGB from 1988 to 2001 (Stock et al 2008). Both 
cohorts were comparable for the main clinical and biologic characteristics, although the median age of the patients in the CALGB studies was $19 \mathrm{yr}$. compared to $16 \mathrm{yr}$. for the CCG patients. CR rates were identical $(90 \%)$ for both the CALGB and CCG AYA. The CCG AYA had a $63 \%$ EFS and $67 \%$ overall survival (OS) probabilities at 7 years in contrast to the CALGB AYA, in whom the 7 -year EFS was only $34 \%$ and the OS was $46 \%$. While the CALGB AYA aged 16 to 17 years achieved similar outcomes to all the CCG AYA with a 7year EFS of $55 \%$, the EFS for 18- to 20 -year-old CALGB patients was only $29 \%$. CALGB AYAs had a significant increase in CNS relapse (11\%) compared to CCG AYAs $(1.5 \%)$. Comparison of the regimens showed that the CCG AYA received earlier and more intensive and prolonged CNS prophylaxis and higher cumulative doses of nonmyelosuppressive agents (vinca alkaloid, steroids and asparaginase), as well as longer duration of maintenance therapy than CALGB AYAs. There were no differences in outcomes in those who reached maintenance therapy on time compared with those who were delayed.

A similar Dutch study in patients aged 15-21 yr yielded similar results (de Bont et al 2004), with a 5-yr EFS of $69 \%$ for comparable patients treated with the more dose-intensive pediatric protocol DCOG vs. $34 \%$ for those treated with adult protocols ALL-5 and ALL-18 from the HOVON Group. Likewise, comparative retrospective studies from Italy also showed a poorer prognosis for patients aged 14-18 yr treated with adult-type protocols (Testi et al 2004). In turn, a Swedish study compared patients aged 10-40 yr treated with the pediatric trial NOPHO-92 $(\mathrm{n}=144)$ vs. a similar group of patients included in the Swedish Adult ALL Group ( $n=99$ ) (Hallbook et al 2006). A significantly higher CR rate (99\% vs. 90\%) and EFS were observed in patients treated with the pediatric protocol, with the type of treatment being an independent prognostic variable on multivariate analysis. However, it is of note that adults aged 26-40 yr had a significantly poorer prognosis than AYA (15-25 yr.). Another study from Denmark yielded similar results (Schroeder et al 2006). In a retrospective study from the British Medical Research Council (MRC) performed only in adolescents (15-17 yr) included in the ALL97/revised99 (pediatric, $n=61)$ or UKALLXII/E2993 (adult, $\mathrm{n}=67$ ) trials between 1997 and 2002 (Ramanujachar et al 2007), the EFS (65\% vs. $49 \%)$ was higher and the rate of death in remission was lower in the former group of patients. In a retrospective study from the Princess Margaret Hospital from Toronto restricted to AYA with T-ALL 40 patients (median age $30 \mathrm{yr}$, range 17-69) were treated with several adult type protocols and were compared with 32 patients (median age $32 \mathrm{yr}$, range 17-64) treated with a DFCI protocol (Al-Khabori et al 2010). Although there were no differences in CR attainment (93\% vs. $84 \%$ ), the OS and relapse-free survival (RFS) probabilities were significantly higher in patients treated with the DFCI trial ( $83 \%$ vs $.56 \%$ and $88 \%$ vs. $23 \%$, respectively). On multivariate analysis the treatment group (DFCI vs. nonDFCI) was the major prognostic factor influencing both RFS and OS. Other studies from different countries (Lopez-Hernandez et al 2008) have shown similar results (Table 1).

Only one population-based study from Finland showed that the outcome of AYA with ALL treated with pediatric or adult protocols was comparable (Usvasalo et al 2008). One hundred and twenty-eight patients (10-16 $\mathrm{yr}$, median age $12.9 \mathrm{yr})$ were treated with the pediatric Nordic (NOPHO) protocols and 97 patients (17-25 yr, median age $18.9 \mathrm{yr}$ ) with Finnish Leukemia Group National protocols. All patients were centrally referred and treated in five academic centers. The 5 -year EFS was $67 \%$ for the pediatric treatment group and $60 \%$ for the adult treatment group. There were no significant differences in the cumulative doses of corticosteroids, vincristine and asparaginase between pediatric and adult protocols, although pediatric protocols used a higher cumulative dose of methotrexate and lower 


\begin{tabular}{|l|l|c|c|c|c|}
\hline Country (reference) & Protocol & Age (yr) & N & CR (\%) & EFS (\%) \\
\hline USA (32) & CCG(P) & $16-20$ & 197 & 90 & 63 \\
\hline & CALGB(A) & & 124 & 90 & 34 \\
\hline France (12) & FRALLE93(P) & $15-20$ & 77 & 94 & 67 \\
\hline Holland (13) & LALA94 (A) & & 100 & 83 & 41 \\
\hline & DCOG (P) & $15-18$ & 47 & 98 & 69 \\
\hline Italy (35) & HOVON (A) & & 44 & 91 & 34 \\
\hline & AIEOP (P) & $14-18$ & 150 & 94 & 80 \\
\hline Sweden (11) & GIMEMA (A) & & 95 & 89 & 71 \\
\hline & NOPHO-92(P) & $10-40$ & 144 & 99 & 65 \\
\hline UK (25) & Adult (A) & & 99 & 90 & 48 \\
\hline & ALL97 (P) & $15-17$ & 61 & 98 & 65 \\
\hline Canada (1) & UKALLXII(A) & & 67 & 94 & 49 \\
\hline & DFCI (P) & $17-64$ & 32 & 84 & $83^{*}$ \\
\hline Mexico (16) & Adult (A) & $17-69$ & 40 & 93 & $56^{*}$ \\
\hline & LALIN (P) & $15-25$ & 20 & 90 & 70 \\
\hline Finland (38) & LALA (A) & & 20 & 80 & 40 \\
\hline & NOPHO (P) & $10-25$ & 128 & 96 & 67 \\
\hline
\end{tabular}

$\mathrm{N}$ : number of patients; CR: complete remission. EFS: event-free survival.

* Overall survival

Table 1. Retrospective comparative studies in adolescents and young adults with acute lymphoblastic leukemia treated with pediatric-based (P) vs. adult-based (A) protocols.

doses of anthracyclines than adult protocols; epipodophyllotoxins and mitoxantrone were not included in the pediatric protocols. The authors attributed the similar results to the similarity of the pediatric and adult protocols and to the centralized care of the patients in five academic centers, ensuring good compliance and adherence to the protocols. Finally, the retrospective data from the MD Anderson Cancer Center using the Hyper-CVAD regimen (not including asparaginase) have also reported favorable results in 102 AYA (median age $19 \mathrm{yr}$ ), with CR $97 \%$ and OS 65\% (Thomas et al 2008). Preliminary reports from 60 AYA patients aged 12-40 yr treated at the MD Anderson Cancer Center with modified augmented Berlin-Frankfurt-Münster (BFM) therapy showed very promising results (2-yr DFS and OS probabilities of $85 \%$ and $91 \%$, respectively) in the subset of patients younger than 25 yr (Rytting et al 2010), stressing the importance of treating these patients in large referral centers.

In summary, the 5- to 6-yr EFS rate for AYA treated with pediatric regimens ranges from $65 \%$ to $70 \%$ vs. $35 \%$ to $50 \%$ for adult regimens in almost but not all retrospective comparative studies. However, it is of note that these studies have mainly focused on patients aged 15-21 years, but few have evaluated the results in young adults up to 30 years or more, in whom the frequency of adverse prognostic factors is progressively increasing.

The reasons for the better results of pediatric protocols are multiple (Stock 2010). The first and probably the most important reason lay in the protocol itself. The dose-intensity and the dose-density of the key chemotherapeutic agents for ALL are clearly higher in pediatric protocols. This is especially relevant for drugs such as vincristine (usually capped to $2 \mathrm{mg}$ in adult protocols), glucocorticoids, asparaginase and methotrexate. Conversely, pediatric- 
based protocols include lower doses of alkylating agents, high-dose cytarabine and antracyclines than adult trials. In addition CNS prophylaxis is more intense and prolonged in pediatric regimens than in adult trials. Most of the pediatric protocols include delayed intensifications and an extended maintenance chemotherapy phase, the former being omitted in many adult trials. The use of allogeneic stem cell transplantation (SCT) as part of first line therapy (associated with a transplant-related mortality [TRM] of 20\%), is restricted to patients with very high-risk features in pediatric trials, whereas it is more widely used in adult trials, even in standard-risk patients in first CR.

The second reason is the tolerability to essential drugs such as asparaginase, steroids and vincristine, which is poorer in AYA compared to children, being a reflection of changes in the metabolism of these drugs during late adolescence. The incidences of diabetes mellitus, pancreatitis, thrombosis and osteonecrosis are more frequent in the former group. The increased toxicity influences adherence to treatment, which is critical for the outcome of ALL patients.

The third reason is the disparity in the practice patterns of the pediatric and adult hematologists/oncologists and patient compliance. Adherence to treatment is usually higher in pediatric than in adult-derived studies, probably due both to a highly skilled supportive staff, the better tolerability of pediatric protocols and a stricter control of time points of chemotherapy delivery in pediatric than in adult hematology units (Burke et al 2007) However, in the U.S. and Canada most children and adolescents with ALL treated with pediatric protocols are managed in institutions and academic centers participating in national-sponsored clinical trials, whilst most AYA treated with adult protocols are managed throughout study groups by community-based medical oncologists. The fact that the most striking differences are observed in the 18 to 20 -years-old group could be explained by the emancipation of some of these patients from parental control and support and the possible need to face significantly more challenges in access to health care due to insurance issues (Kantarjian et al 2009).

\subsection{Prospective trials}

\subsubsection{Results of the treatment of adolescents in pediatric trials}

Barry et al reported the outcome of adolescents treated in the Dana-Farber Cancer Institute (DFCI) ALL Consortium Protocols conducted between 1991 and 2000 (Barry et al 2007). A total of 844 patients aged 1 to 18 years, with newly diagnosed ALL were enrolled into two consecutive DFCI-ALL Consortium Protocols. Outcomes were compared in three age groups: children aged 1 to 10 years $(n=685)$, young adolescents aged 10 to 15 years $(n=108)$, and older adolescents aged 15 to 18 years $(n=51)$. With a median follow-up of 6.5 years, the 5-year EFS for those aged 1 to 10 years was $85 \%$, compared with $77 \%$ for those aged 10 to 15 years, and $78 \%$ for those aged 15 to 18 years. There was no difference in the rate of treatment-related complications between the 10- to 15-year and 15- to 18-year age groups.

Nachman et al reported the results of the CCG1961 trial including AYA up to $21 \mathrm{yr}$ (Nachman et al 2009). The EFS and overall survival (OS) rates were $71.5 \%$ and $77.5 \%$, respectively. Rapid responder patients randomly assigned to augmented therapy had 5-year EFS $81.8 \%$ vs $66.8 \%$ for patients receiving standard therapy, but 1 versus 2 interim maintenance and delayed intensification courses had no significant impact on EFS. WBC count over $50 \times 10^{9} / \mathrm{L}$ was an adverse prognostic factor. Given the excellent outcome with this chemotherapy there seems to be no role for the routine use of stem cell transplantation in first remission 
In turn, the results of the total therapy studies XIIIA, XIIIB, XIV and XV from St Jude Children's Research Hospital including 963 pediatric patients, 89 of whom were older adolescents (aged 15 to $18 \mathrm{yr}$.), have recently been published (Pui et al 2011). In the first three studies the 44 older adolescents had significantly poorer EFS and OS than the 403 younger patients. On the contrary, in study XV (incorporing the level of MRD to guide treatment, with featured intensive methotrexate, vincristine, glucocorticoid and asparaginase and early triple intrathecal chemotherapy for higher risk ALL) the EFS of 45 older adolescents was $86.4 \%$, similar to $87.4 \%$ for the 453 younger children. The OS was also comparable $(87.9 \%$ vs. $94.1 \%$, respectively). The authors concluded that most older adolescents with ALL can be cured with risk-adjusted intensive chemotherapy without SCT. In summary, with modern approaches of treatment of ALL with pediatric-based protocols the unfavorable prognosis of adolescents is disappearing and hopefully, this improvement could be translated to young adults.

\subsubsection{Results of prospective studies in adolescent and young adults}

Some studies have evaluated or are currently evaluating the feasibility and results of the pediatric-based protocols administered to adults up to 30 or even up to 50 or 60 years of age (table 2). The Spanish PETHEMA group compared the results of the pediatric protocol ALL96 in adolescents $(15-18 \mathrm{yr}, \mathrm{n}=35)$ and young adults $(18-30 \mathrm{yr}, \mathrm{n}=46)$ with standard-risk (SR) ALL (Ribera et al 2008). Both groups were comparable for the main clinical and biologic characteristics of ALL. The CR rate was $98 \%$ and after a median follow-up of 4.2 yr., 6-year EFS and OS were $61 \%$ and $69 \%$, with no differences between adolescents and young adults. No significant differences were observed in the timing of treatment delivery, although the hematologic toxicity in consolidation and reinforcement cycles was higher in young adults than in adolescents. These results suggest that pediatric protocols can be effectively and safely employed in adult patients with SR ALL, at least up to the age of $30 \mathrm{yr}$.

\begin{tabular}{|l|l|c|c|c|c|}
\hline Country (reference) & Protocol & Age (yr) & N & CR (\%) & EFS (\%) \\
\hline Spain (26) & PETHEMA ALL-96 & $15-18$ & 35 & 94 & 60 \\
\hline & & $19-30$ & 46 & 100 & 63 \\
\hline France (32) & GRAALL-2003 & $15-45$ & 172 & 95 & 58 \\
\hline USA (6) & DFCI & $18-50$ & 74 & 82 & $72.5^{* *}$ \\
\hline Canada (34) & Modified DFCI & $17-71$ & 68 & 85 & $65^{* * *}$ \\
\hline France (10) & FRALLE2000 & $18-55$ & 40 & 90 & $72^{* * *}$ \\
\hline
\end{tabular}

* Results restricted to adolescents; ${ }^{* *}$ Estimated at 2 years; ${ }^{* * *}$ Overall survival.

$\mathrm{N}$ : number of patients; CR: complete remission. EFS: event-free survival.

Table 2. Prospective studies in adolescents and young adults with acute lymphoblastic leukemia treated with pediatric-based or inspired protocols 
The French GRAALL group has reported the results of the pediatric-inspired GRAALL-2003 study including 215 patients aged 15-60 yr (Huguet et al 2009). In this study there was an 8.6-fold, 3.7-fold and 16-fold increase in cumulative doses of prednisone, vincristine and asparaginase, respectively, compared with the previous adult-based LALA-94 protocol, although the GRAALL-2003 trial retained some adult options, such as allogeneic SCT for patients with high-risk ALL. The CR rate was $93.5 \%$ and at 42 months the EFS and overall survival (OS) rates were $55 \%$ and $60 \%$, respectively. The CR rate, EFS and OS compared favorably with the previous LALA-94 experience. It is of note, however, that in patients over $45 \mathrm{yr}$ there was a higher cumulative incidence of chemotherapy-related deaths $(23 \% \mathrm{vs}$. $5 \%)$ and deaths in first CR (22\% vs. $5 \%)$, although the incidence of relapse remained stable (30 vs. $32 \%$ ). The results of this study suggest that pediatric-inspired therapy is feasible in young adults with ALL at least until the age of $45 \mathrm{yr}$, in whom the outcome clearly improves.

Based on the promising results obtained in adolescents with ALL, the DFCI Combined Adult/Pediatric ALL Consortium has applied a true pediatric protocol to adults aged 18-50 yr (DeAngelo et al 2006). Specifically, the investigators used an extended course of asparaginase for 30 weeks. The preliminary results in 94 patients, with a median age of $28 \mathrm{yr}$ have shown a CR in 79 patients (84\%). With a median follow-up of 45 months, the estimated DFS rate was $66 \%$ and the OS rate was $68 \%$. This study proved that extended asparaginase treatment was feasible in adults and the drug-related toxicity was manageable, although the incidence of pancreatitis (13\%) and thrombosis/embolism (19\%) was a matter of concern. In turn, the Princess Margaret Hospital used a modified Dana Farber Cancer Institute pediatric protocol in 68 adult patients ( 17 to $71 \mathrm{yr}$ ), with a CR rate of $85 \%$ and 3 -yr OS and DFS of $65 \%$ and $77 \%$, respectively (Storring et al 2009). The University of South California group (Srivastava et al 2008) used an augmented BFM pediatric regimen with eight doses of pegylated asparaginase to treat adults with ALL aged 19-57 yr (median 33), with a 3-yr projected EFS of $65 \%$. Toxicity attributable to asparaginase was frequent but manageable. However, older patients had significantly less tolerance to asparaginase, vincristine and steroids compared to children or adolescents. In the FRALLE group from France 28 Philadelphia chromosome-negative adult ALL patients 16 to 57 years of age were treated in the FRALLE 2000 protocol consisting of a prednisone pre-phase and a four-drug induction including asparaginase, consolidation, delayed intensification and maintenance chemotherapy. The 4 -yr DFS was $90 \%$ vs. $47 \%$ seen in matched historical controls (Haiat et al 2011).

The largest prospective phase II trial to evaluate the feasibility of the use of a true pediatric regimen in AYA is currently ongoing in the US (C-10403 trial) (available at www.clinicaltrials.gov. NCT00558519) AYAs from 16 to $39 \mathrm{yr}$ are treated by adult oncologists/hematologists with one treatment arm of the current AALL0232 trial from the pediatric COG group. More than half of the 300 planned patients have been currently enrolled. This study will prospectively analyze the genetic characteristics, MRD, treatment adherence, tolerability and psycho-social and socio-economic conditions that likely influence treatment outcomes.

The results from these prospective studies demonstrate the feasibility and tolerability of pediatric-based regimens in AYA with SR ALL, at least until 30-50 yr. All these trials have the increased cumulative dosages of the most important drugs for ALL therapy, the cautious observance of dose-intensity and the reduction of the delays between the different phases of the therapy in common. If these results can be confirmed with a longer follow-up, they will 
have an impact on the clinical management of AYA patients in the future. Finally, few specific data are available on long-term complications of successful treatment of AYA (see www.survivorshipguidelines.org by the COG group) and a comprehensive approach to the follow-up for this significant group of patients with ALL is lacking. The next generation of studies, incorporating biological, pharmacological and psychosocial issues will further improve the cure rate and quality of life of these patients.

\section{Acknowledgment}

Supported in part by grants RD06/0020/1056 from RTICC, Instituto Carlos III, PI051490 from Fondo de Investigaciones Sanitarias and FIJC P/EF-10 from Jose Carreras Leukemia Foundation.

\section{References}

[1] Al-Khabori M, Minden MD, Yee KWL, et al. Improved survival using an intensive, pediatric-based chemotherapy regimen in adults with T-cell acute lymphoblastic leukemia. Leuk \& Lymphoma. 2010; 51: 61-65.

[2] Barry E, DeAngelo DJ, Neuberg D, et al. Favorable outcome for adolescents with acute lymphoblastic leukemia treated on Dana Farber Cancer Institute ALL Consortium protocols. J Clin Oncol. 2007; 25: 813-819.

[3] Bleyer A, O'Leary M, Barr R, Ries LAG (eds). Cancer Epidemiology in Older Adolescents and Young Adults 15 to 29 years of Age, including SEER Incidence and Survival: 1975-2000. National Cancer Institute, NIH Pub. No. 06-5767. Bethesda, MD 2006.

[4] Boissel N, Auclerc M-F, Lheritier V, et al. Should adolescents with acute lymphoblastic leukemia be treated as old children or young adults? Comparison of the French FRALLE-93 and LALA-94 trials. J Clin Oncol. 2003;21:774-780.

[5] Burke ME, Albriton K, Marina N. Challenges in the recruitment of adolescents and young adults to cancer clinical trials. Cancer. 2007; 110: 2385-93.

[6] DeAngelo DJ, Silverman LB, Couban S, et al. A multicenter phase II study using a dose intensified pediatric regimen in adults with untreated acute lymphoblastic leukaemia. Blood. 2006; 108: 526a

[7] De Bont JM, van der Holt B, Dekker AW, van der Does-van den Berg A, Sonneveld P, Pieters R. Significant difference in outcome for adolescents with acute lymphoblastic leukemia treated on pediatric vs adult protocols in the Netherlands. Leukemia. 2004;18: 2032-2035.

[8] Gokbuget N, Hoelzer D. Treatment of adult acute lymphoblastic leukemia. Semin Hematol. 2009; 46: 64-75.

[9] Haiat S, Marjanovic Z, Lapusan S, et al. Outcome of 40 adults aged from 18 to 55 years with acute lymphoblastic leukemia treated with double delayed intensification pediatric protocol. Leuk Res. 2011;35: 66-72.

[10] Hallbook H, Gustafsson G, Smedmyr B, Soderhall S, Heyman M; Swedish Adult Acute Lymphocytic Leukemia Group; Swedish Childhood Leukemia Group. Treatment outcome in young adults and children $>10$ years of age with acute lymphoblastic leukemia in Sweden: a comparison between a pediatric protocol and an adult protocol. Cancer. 2006;107:1551-61. 
[11] Huguet F, Raffoux E, Thomas X, et al. Pediatric inspired therapy in adults with Philadelphia chromosome-negative acute lymphoblastic leukemia: the GRAALL/2003 study. J Clin Oncol. 2009; 27: 911-8.

[12] Juliusson, G, Karlsson K, Hallböök H. Population-based analyses in adult acute lymphoblastic leukemia. Blood. 2010;116:1011.

[13] Kantarjian HM, O'Brien S. Insurance policies in the United States may explain part of the outcome differences of adolescents and young adults with acute lymphoblastic leukemia treated on adult versus pediatric regimens. Blood. 2009; 113: 1861.

[14] Larson R, Stock W. Progress in the treatment of adults with acute lymphoblastic leukemia. Curr Opin Hematol .2008; 15: 400-7.

[15] Lopez-Hernandez MA, Alvarado-Ibarra M, Jiménez-Alvarado RM, De Diego-Flores JE, González-Avante CM. Adolescents with de novo acute lymphoblastic leukemia: efficacy and safety of a pediatric vs. adult treatment protocol. Gac Med Mex. 2008; 144: 485-9.

[16] Moorman AV, Chilton L, Wilkinson J, et al. A population-based cytogenetic study of adults with acute lymphoblastic leukemia. Blood. 2010;115:206-214.

[17] Moorman AV, Ensor HM, Richards SM, et al. Prognostic effect of chromosomal abnormalities in childhood B-precursor acute lymphoblastic leukaemia: results from the UK Medical Research Council ALL 97/99 randomised trial. Lancet Oncol. 2010; 11: 429-438.

[18] Nachman JB, La MK, Hunger SP, et al. Young Adults With Acute Lymphoblastic Leukemia Have an Excellent Outcome With Chemotherapy Alone and Benefit From Intensive Postinduction Treatment: A Report From the Children's Oncology Group. J Clin Oncol.2009; 27:5189-5194

[19] Pui CH, Robison LL, Look AT. Acute lymphoblastic leukaemia. Lancet. 2008; 371:103043.

[20] Pui CH, Pei D, Campana D, et al. Improved prognosis for older adolescents with acute lymphoblastic leukemia. J Clin Oncol. 2011; 29: 386-391.

[21] Pullen J, Shuster JJ, Link M, et al. Significance of commonly used prognostic factors differs for children with T cell acute lymphocytic leukemia (ALL), as compared to those with B-precursor ALL. A Pediatric Oncology Group (POG) study. Leukemia. 1999; 13:1696-1707.

[22] Pulte D, Gondos A, Brenner H. Trends in 5-and 10-year survival after diagnosis with childhood hematologic malignancies in the United States 1990-2004. J Natl Cancer Inst. 2008; 100: 1271-3.

[23] Pulte D, Gondos A, Brenner H. Trends in survival after diagnosis with hematologic malignancy in adolescence and young adulthood in the United States, 1981-2005. Cancer. 2009; 115: 4973-4979.

[24] Ramanujachar R, Richards S, Hann I, Webb DD. Adolescents with acute lymphoblastic leukaemia: outcome on UK national paediatric (ALL97) and adult (UKALLXII/E2993) trials. Pediatr Blood Cancer. 2007;48:254-61.

[25] Ribera JM, Oriol A, Sanz MA, et al. Comparison of the results of the treatment of adolescents and young adults with standard-risk acute lymphoblastic leukemia with the pediatric-based protocol PETHEMA ALL-96. J Clin Oncol. 2008; 26:1843-9. 
[26] Rytting ME, Kantarjian HM, Thomas ED, et al. Adolescent and Young Adult Patients Treated with Modified Augmented Berlin-Frankfurt-Muenster Therapy [abstract]. Blood. 2010; 114

[27] Schroder H, Kjeldahl M, Boesen AM, et al. Acute lymphoblastic leukemia in adolescents between 10 and 19 years of age in Denmark. Dan Med Bull. 2006;53:769.

[28] Secker-Walker LM, Craig JM, Hawkins JM, Hoffbrand AV. Philadelphia positive acute lymphoblastic leukemia in adults: age distribution, BCR breakpoint and prognostic significance. Leukemia. 1991;5:196-199.

[29] Smith MA, Siebel NL, Altefruse SF, et al. Outcomes of children and adolescents with cancer: challenges for the twenty-first century. J Clin Oncol. 2010; 28:2625-2634.

[30] Srivastava P, Watkins K, Mark L, et al. Treatment of adults with newly diagnosed acute lymphoblastic leukemia with multiple doses of intravenous pegylated asparaginase in an intensified pediatric regimen. Haematologica. 2008; 93 (s1): 366.

[31] Stock W, La M, Sanford B, et al. What determines the outcomes for adolescents and young adults with acute lymphoblastic leukemia treated on cooperative protocols? A comparison of Children's Cancer Group and Cancer and Leukemia Group B studies. Blood. 2008;112:1646-74.

[32] Stock W. Adolescents and young adults with acute lymphoblastic leukemia. Hematology 2010. American Society of Hematology Education Program Book. 2010; 21-29.

[33] Storring JM, Minden MD, Kao S, et al. Treatment of adults with BCR-ABL negative acute lymphoblastic leukaemia with a modified paediatric regimen. Br J Haematol. 2009; 146: 76-85.

[34] Testi AM, Valsecchi MG, Conter V, et al. Difference in outcome of adolescents with acute lymphoblastic leukemia (ALL) enrolled in pediatric (AIEOP) and adult (GIMEMA) protocols. Blood. 2004;104:1954a

[35] Thomas DA, O'Brien S, Rytting M, et al. Acute lymphoblastic leukemia (ALL) or lymphoblastic lymphoma (LL) after frontline therapy with hyper-CVAD regimens [abstract]. Blood. 2008; 112: 674.

[36] Usvasalo A, Räty R, Knuutila S, et al. Acute lymphoblastic leukemia in adolescents and Young adults in Finland. Haematologica. 2008; 93: 1161-8.

[37] Available at www.clinicaltrials.gov NCT00558519. 


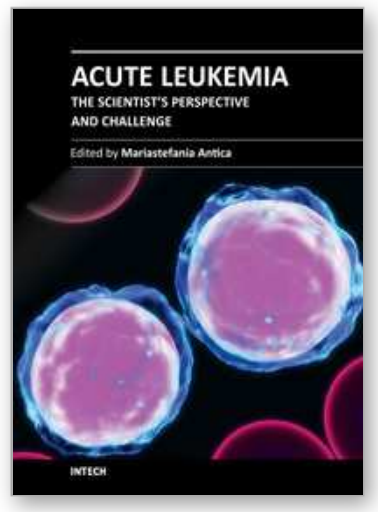

\author{
Acute Leukemia - The Scientist's Perspective and Challenge \\ Edited by Prof. Mariastefania Antica
}

ISBN 978-953-307-553-2

Hard cover, 428 pages

Publisher InTech

Published online 22, December, 2011

Published in print edition December, 2011

This book provides a comprehensive overview of he basic mechanisms underlying areas of acute leukemia, current advances, and future directions in management of this disease. The first section discusses the classification of acute leukemia, taking into account diagnoses dependent on techniques that are essential, and thankfully readily available, in the laboratory. The second section concerns recent advances in molecular biology, markers, receptors, and signaling molecules responsible for disease progression, diagnostics based on biochips and other molecular genetic analysis. These advances provide clinicians with important understanding and improved decision making towards the most suitable therapy for acute leukemia. Biochemical, structural, and genetic studies may bring a new era of epigenetic based drugs along with additional molecular targets that will form the basis for novel treatment strategies. Later in the book, pediatric acute leukemia is covered, emphasizing that children are not small adults when it comes to drug development. The last section is a collection of chapters about treatment, as chemotherapy-induced toxicity is still a significant clinical concern. The present challenge lies in reducing the frequency and seriousness of adverse effects while maintaining efficacy and avoiding over-treatment of patients.

\title{
How to reference
}

In order to correctly reference this scholarly work, feel free to copy and paste the following:

Josep-Maria Ribera (2011). Acute Lymphoblastic Leukemia in Adolescents and Young Adults, Acute Leukemia - The Scientist's Perspective and Challenge, Prof. Mariastefania Antica (Ed.), ISBN: 978-953-307-553-2, InTech, Available from: http://www.intechopen.com/books/acute-leukemia-the-scientist-s-perspective-andchallenge/acute-lymphoblastic-leukemia-in-adolescents-and-young-adults

\section{INTECH}

open science / open minds

\section{InTech Europe}

University Campus STeP Ri

Slavka Krautzeka 83/A

51000 Rijeka, Croatia

Phone: +385 (51) 770447

Fax: +385 (51) 686166

www.intechopen.com

\section{InTech China}

Unit 405, Office Block, Hotel Equatorial Shanghai

No.65, Yan An Road (West), Shanghai, 200040, China

中国上海市延安西路 65 号上海国际贵都大饭店办公楼 405 单元

Phone: +86-21-62489820

Fax: +86-21-62489821 
(C) 2011 The Author(s). Licensee IntechOpen. This is an open access article distributed under the terms of the Creative Commons Attribution 3.0 License, which permits unrestricted use, distribution, and reproduction in any medium, provided the original work is properly cited. 